\title{
Implant-Associated Infection of Long-Segment Spinal Instrumentation: A Retrospective Analysis of 46 Consecutive Patients
}

\author{
Stavros Oikonomidis, Lisa Altenrath, Leonard Westermann, Jan Bredow, Peer Eysel, Max Joseph Scheyerer \\ Department of Orthopedics and Trauma Surgery, Faculty of Medicine and University Hospital Cologne, University of Cologne, Cologne, Germany
}

Study Design: This single-center retrospective study analyzed patients with an implant-associated infection of spinal instrumentation (four or more segments) treated between 2010 and 2018.

Purpose: This study aimed to investigate the treatment of implant-associated infections of long-segment spinal instrumentation and to define risk factors for implant removal.

Overview of Literature: Implant-associated infection occurs in $0.7 \%-20 \%$ of spinal instrumentation. Significant blood loss, delayed reoperation, and use of effective antibiotics are reported risk factors for implant removal.

Methods: Patients with superficial infections not involving the implant were excluded. All patients received surgical and antibiotic treatments according to our interdisciplinary osteomyelitis board protocol. An infection was considered healed if a patient showed no signs of infection 1 year after termination of treatment. The patients were divided into an implant retention group and implant removal group, and their clinical and microbiological data were compared.

Results: Forty-six patients (27 women, 19 men) with an implant-associated infection of long-segment spinal instrumentation and mean age of $65.3 \pm 14.3$ years (range, 22-89 years) were included. The mean length of the infected instrumentation was $6.5 \pm 2.4$ segments (range, 4-13 segments). Implant retention was possible in 21 patients (45.7\%); in the other 25 patients (54.3\%), a part of or the entire implant required removal. Late infections were associated with implant removal, which correlated with longer hospitalization. Both groups showed high postoperative complication rates $(50 \%)$ and high mortality rates $(8.7 \%)$. In 39 patients $(84.8 \%)$, infection

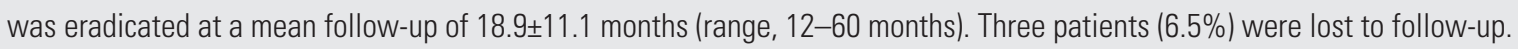

Conclusions: Implant-associated infections of long-segment spinal instrumentations are associated with high complication and mortality rates. Late infections are associated with implant removal. Treatment should be interdisciplinary including orthopedic surgeons and clinical infectiologists.

Keywords: Spinal infection; Implant-associated infection; Long-segmental spinal instrumentation; Deep surgical site infection

\section{Introduction}

Spinal instrumentation is an established treatment for many diseases of the spine such as degenerative conditions, tumors, trauma, and deformity. Infection of spinal instrumentation is a challenging complication that re-

Received Dec 21, 2019; Revised Mar 1, 2020; Accepted Mar 26, 2020

Corresponding author: Stavros Oikonomidis

Department of Orthopedics and Trauma Surgery, Faculty of Medicine and University Hospital Cologne, University of Cologne, Kerpener Str. 62, 50937 Cologne, Gemany

Tel: +49-1724254544, Fax: +49-221-478 6060, E-mail: Stavros.oikonomidis@uk-koeln.de 
quires appropriate surgical and antibiotic treatment. The reported incidence of implant-associated infection of spinal instrumentation ranges from $0.7 \%$ to $20 \%$ [1]. Risk factors include older age, prior surgery, diabetes mellitus, smoking, alcohol abuse, overweight status, malnutrition, extended operation time, and prolonged hospital stay [24]. Postoperative infections are divided into early and late infections. Early infections are commonly caused by virulent bacteria such as Staphylococcus aureus and betahemolytic Streptococcus, while late infections are caused by less virulent bacteria such as Propionibacterium acnes and Staphylococcus epidermidis [5].

The treatment of implant-associated spinal infections includes surgical debridement and irrigation, implant removal, vacuum therapy, and long-term antibiotic therapy [6]. The spine is in a relatively hypoxic environment, and antibiotics have attenuated efficacy against implant-associated bacteria [7]. In addition, the formation of bacterial biofilm on the spinal hardware can lead to a persistent microbiological infection despite proper antibiotic treatment [8]. Accordingly, implant removal is recommended by some authors [9-11]. However, removal of long-segment instrumentation can lead to progressive deformity, pain, and pseudarthrosis [12]. These aspects suggest the challenging nature of treatment of implant-associated infection of spinal instrumentation. Moreover, no firm recommendations have been established.

To the best of our knowledge, the influence of instrumentation length on treatments and outcomes of implantassociated spinal infections has not been investigated. Thus, this study aimed to investigate treatment of implant-associated infections of long-segment spinal instrumentation in a high-volume institution, to recommend surgical and antibiotic treatment strategies, and to determine risk factors for implant removal in long-segment instrumentation.

\section{Materials and Methods}

\section{Study design}

This single-center retrospective study analyzed data of all consecutive patients with an implant-associated infection after long-segmental spinal instrumentation from January 1,2010 to January 2, 2018. This study was approved by the Institutional Review Board of the Faculty of Medicine and University Hospital Cologne (approval no., 19-1008). All patients were treated according to the recommendations of the institutional interdisciplinary osteomyelitis board, which includes orthopedic surgeons and clinical infectiologists. All procedures performed in the study involving human participants were in accordance with the 1964 Helsinki Declaration and its later amendments or comparable ethical standards.

The inclusion criteria were deep infection after longsegment spinal instrumentation (four segments or more), treatment protocol of the osteomyelitis board, and complete follow-up (at least 1 year after termination of treatment). The exclusion criteria were superficial infections of the wound and previous history of vertebral osteomyelitis.

To determine risk factors for implant removal, patients were divided into the implant retention group and implant removal group (partial or complete).

\section{Data collection}

Patient's electronic medical records were reviewed, and medical history and surgical, clinical, and microbiological data were recorded and assessed. Indications for spinal instrumentation were degeneration, trauma, tumor, or deformity.

\section{Clinical and laboratory data}

Clinical symptoms such as back pain, radiculopathy, fever, neurological deficits, and infection signs (redness, fistulation, hyperthermia, swelling, drainage) on the wound/ operation site were assessed. Early infections were defined as symptoms that started earlier than 3 months after the index/last surgery, and late symptoms started 3 months after the index/last surgery [1].

In addition, the American Society of Anesthesiologists (ASA) classification, pre-existing conditions (risk factors), length of hospital stay, length of intensive care unit stay, perioperative and postoperative complications, duration of intravenous and oral antibiotic therapy, and readmission rate due to recurrent infection were assessed. Complications were classified as general and surgical complications. Laboratory parameters C-reactive protein (CRP) and white blood cell (WBC) count were documented upon admission.

Instrumentation location was categorized as cervical, thoracic, lumbar, sacral, or a combination thereof. Instrumentation length was measured as the number of segments. 


\section{Surgical treatment of implant-associated infection}

The number of surgical interventions required to eradicate an infection was recorded. Moreover, the surgical intervention method was documented and classified as debridement only and partial or complete implant removal with or without re-instrumentation. Outcomes of wound closure methods such as vacuum-assisted closure (VAC) or overlap plastic were assessed. Debridement was defined as wide surgical debridement of soft tissue, removal of loose or necrotic bone tissue, extended irrigation with at least $6 \mathrm{~L}$ of sodium chloride solution, and primary wound closure over suction drains.

Implant removal was performed in cases of implant loosening detected by radiological examinations (radiography or computed tomography) or persistent infection despite surgical debridement. In persistent infection, the decision to remove the implant was made by the interdisciplinary osteomyelitis board.

\section{Microbiological data}

Microbiological findings of blood culture, intraoperative probe culture, and polymerase probe reaction (by polymerase chain reaction) examination of the intraoperative probe were assessed. The infections were divided into monomicrobial when only one pathogen was isolated and polymicrobial when two or more pathogens were isolated.

In case of an apparent infection or septic condition, calculated antibiosis was administered (flucloxacillin and ceftriaxone) until the results of the intraoperative probes or blood culture probes were known. If the patient was not septic and an infection was not evident, antibiotic treatment was delayed until the pathogen was identified.

The antibiotic therapy was adjusted following identification of the pathogen and in accordance with the resistogram and decision of the osteomyelitis board. In implant retention or re-instrumentation after implant removal, a biofilm-active antimicrobial agent was used instead. The biofilm-active antimicrobial agent was rifampicin $600 \mathrm{mg}$ 1-0-0 or fosfomycin $3 \mathrm{~g} \mathrm{1-0-0}$ for rifampicinresistant pathogen. The antibiotic treatment was given for 3 months.

\section{Terminology}

Infection was defined according to the Centers for Disease
Control and Prevention criteria, which includes a condition with abscess or other evidence of soft tissue infection [13]. The Centers for Disease Control and Prevention defined deep infections as a condition resulting in an abscess or other evidence of infection in deep soft tissue muscle and fascia [13]. An implant-associated infection of the instrumentation was judged as healed in cases with normal laboratory inflammatory markers (CRP level and leukocyte count) and dry wound condition at 1-year follow-up after termination of the treatment.

\section{Statistical analysis}

IBM SPSS ver. 25.0 (IBM Corp., Armonk, NY, USA) was used to evaluate data. Descriptive and frequency analyses were used to describe demographic data, clinical data, and outcomes. The mean values between the two groups were analyzed using the Mann-Whitney test. The median was analyzed for ordinal values such as number of surgical interventions. Cross tables were used to analyze correlations between variables. Contingency coefficient $c$ was used to calculate the correlation between nominal parameters, while eta coefficient $r$ was used to calculate correlations between nominal and metrical parameters. Multiple regression analysis was carried out to determine risk factors for implant removal. Values of $p<0.05$ were considered statistically significant.

\section{Results}

\section{Demographics and clinical results}

During this period, 46 patients developed an implant-associated infection of long-segment spinal instrumentation and met the inclusion criteria: 27 (58.7\%) were women and $19(41.3 \%)$ were men. The mean patient age at the time of infection diagnosis was $65.3 \pm 14.3$ years (range,

Table 1. Distribution of ASA classification

\begin{tabular}{lc} 
Variable & No. $(\%)$ \\
\hline ASA 1 & $2(4.3)$ \\
\hline ASA 2 & $17(37.0)$ \\
\hline ASA 3 & $18(39.1)$ \\
\hline ASA 4 & $9(19.6)$ \\
\hline Total & $46(100.0)$ \\
\hline
\end{tabular}

ASA, American Society of Anesthesiologists. 
22-89 years). As regards the ASA classification, two patients (4.3\%) were classified as ASA 1, 17 patients (37.0\%) as ASA 2, 18 patients (39.1\%) as ASA 3, and nine patients (19.6\%) as ASA 4 (Table 1).

The mean length of the infected instrumentation was $6.5 \pm 2.4$ segments (range, $4-13$ segments). The indication for instrumentation was deformity in six patients, tumor disease in seven, fracture in seven, and degenerative disease in 26. The region of the spinal instrumentation is shown in Table 2. The thoracolumbar region was the most frequently affected site. Among the infections, 32 (69.6\%) were classified as early and $14(30.5 \%)$ were classified as late infections.

At time of admission, 33 patients (71.7\%) had pain at the operated region of the spine, while 34 (73.9\%) showed infection signs such as swelling, secretion, fistulation, and redness. Moreover, 12 patients (26.1\%) had fever,
$17(37.0 \%)$ showed an increased WBC count, and nine (19.6\%) presented neurological deficits. Upon admission,

Table 2. Region with spinal instrumentation

\begin{tabular}{lc} 
Variable & No $(\%)$ \\
\hline Cervical spine & $1(2.2)$ \\
\hline Cervicothoracic spine & $2(4.3)$ \\
\hline Thoracic spine & $3(6.5)$ \\
\hline Thoracolumbar spine & $13(28.3)$ \\
\hline Lumbar spine & $5(10.9)$ \\
\hline Lumbosacral spine & $4(8.7)$ \\
\hline Thoraco-lumbosacral spine & $9(19.6)$ \\
\hline Thoraco-lumbosacral spine-ilium & $8(17.4)$ \\
\hline Lumbosacral spine-ilium & $1(2.2)$ \\
\hline Total & $46(100.0)$ \\
\hline
\end{tabular}

Table 3. Distribution of isolated pathogens in total and between both groups

\begin{tabular}{|c|c|c|c|}
\hline Variable & Total & Group 1 & Group 2 \\
\hline Staphylococcus epidermidis & $20(14 \mathrm{~m}, 4 \mathrm{~b}, 1 \mathrm{t})$ & 9 & 11 \\
\hline Methicillin-susceptible Staphylococcus aureus & $13(\mathrm{~m})$ & 4 & 9 \\
\hline Enterococcus faecalis & $7(3 \mathrm{~m}, 3 \mathrm{~b}, 1 \mathrm{t})$ & 3 & 4 \\
\hline Propionibacterium acnes & $5(3 \mathrm{~m}, 1 \mathrm{~b}, 1 \mathrm{t})$ & 4 & 1 \\
\hline Staphylococcus hominis & $3(1 \mathrm{~m}, 2 \mathrm{~b})$ & 1 & 2 \\
\hline Escherichia coli & $3(2 \mathrm{~m}, 1 \mathrm{~b})$ & 2 & 1 \\
\hline Enterobacter cloacae & $3(1 \mathrm{~m}, 2 \mathrm{t})$ & 3 & 0 \\
\hline Staphylococcus haemolyticus & $2(1 \mathrm{~m}, 1 \mathrm{t})$ & 1 & 1 \\
\hline Corynebacterium tuberculostearicum & $1(\mathrm{~m})$ & 1 & 0 \\
\hline Clostridium perfringens & 1 (b) & 0 & 1 \\
\hline Citrobacter koseri & 1 (b) & 0 & 1 \\
\hline Corynebacterium amycolatum & $1(f)$ & 0 & 1 \\
\hline Corynebacterium jeikeium & 1 (b) & 1 & 0 \\
\hline Staphylococcus lugdunensis & $1(\mathrm{~m})$ & 0 & 1 \\
\hline Enterobacter cloacae 3MRGN & $1(\mathrm{~m})$ & 0 & 1 \\
\hline Finegoldia magna & $1(\mathrm{~m})$ & 1 & 0 \\
\hline Staphylococcus capitis & 1 (b) & 1 & 0 \\
\hline Klebsiella pneumoniae 3MRGN & $1(\mathrm{~m})$ & 0 & 1 \\
\hline Proteus mirabilis & 1 (b) & 0 & 1 \\
\hline Candida albicans & 1 (b) & 0 & 1 \\
\hline Staphylococcus pettenkoferi & 1 (b) & 0 & 1 \\
\hline Vancomycin-resistant Enterococcus & $1(\mathrm{~m})$ & 1 & 0 \\
\hline Pseudomonas aeruginosa & $1(\mathrm{~m})$ & 0 & 1 \\
\hline
\end{tabular}

In the parentheses the distribution of each pathogen in mono-, bi-, tri-, or tetra-microbial is being demonstrated.

m, monomicrobial; b, bi-microbial; t, tri-microbial; $f$, tetra-microbial; MRGN, multi-resistant Gram negative. 
the patients showed a mean CRP level of $137 \pm 106 \mathrm{mg} / \mathrm{L}$ (range, 5-389 mg/L).

\section{Microbiological results}

A monomicrobial infection was diagnosed in 28 patients $(62.2 \%)$, while a polymicrobial infection was diagnosed in 17 patients $(37.8 \%)$. Among the 17 patients, two pathogens were isolated in 10, three pathogens in six, and four pathogens in one patient. In one patient (2.2\%), no pathogen was isolated. The pathogens were isolated in 34 patients $(73.9 \%)$ using the microbiological culture of the intraoperative probe, in six patients (13.0\%) using polymerase chain reaction of the intraoperative probe, in three patients $(6.5 \%)$ using blood cultures, and in two patients (4.3\%) of microbiological culture after sterile puncture of the wound. The detailed distribution of the isolated pathogens is described in Table 3.

\section{Surgical treatment}

All patients were treated surgically. In 21 patients (45.7\%), implant retention was possible; in the other 25 patients $(54.3 \%)$, the entire or a portion of the implant was removed. In implant retention (45.7\%), extensive surgical debridement was performed. In 18 patients (39.1\%), extensive surgical debridement, implant removal, and reinstrumentation were necessary. In five patients (10.9\%), extensive surgical debridement, and partial removal of the implant were performed. In two patients (4.3\%), extensive surgical debridement, and complete implant removal were performed. No radiological changes in the sagittal and coronal planes were observed during follow-up in these two patients; thus, no re-instrumentation was necessary (Fig. 1).
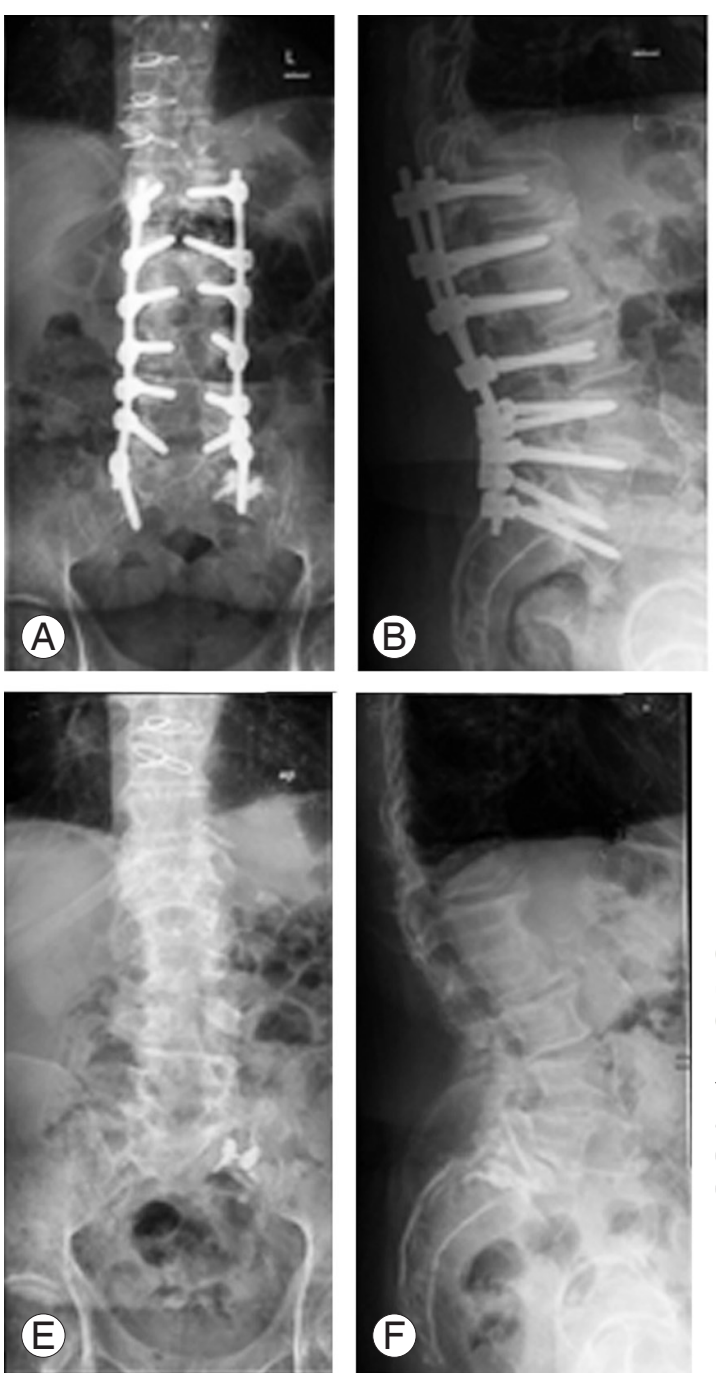
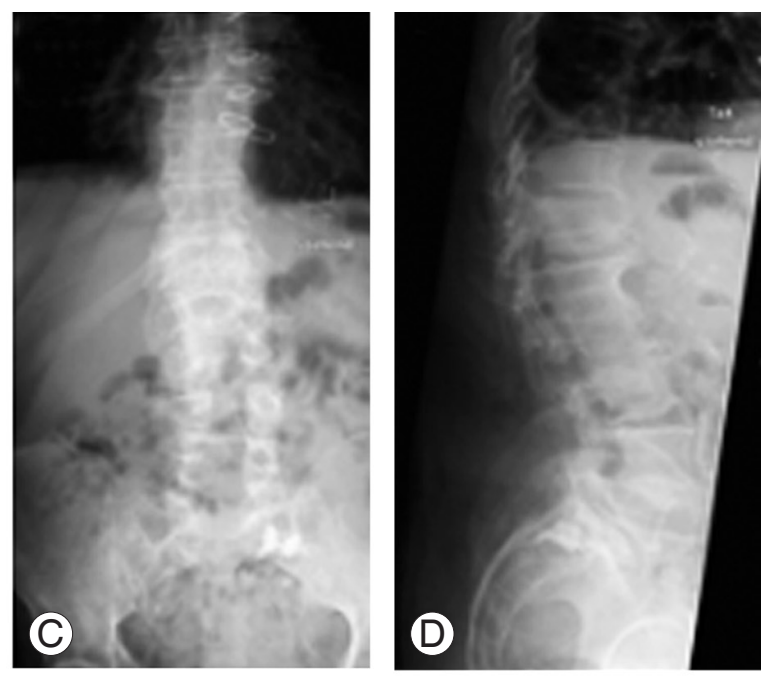

Fig. 1. Radiological examination (X-ray) of the lumbar spine in the anteriorposterior and lateral views in standing position of a patient receiving implant removal but no re-instrumentation due to an implant-associated infection. (A, B) Anterior-posterior and lateral views are the preoperative radiological examinations and demonstrate noticeable pedicle screw loosening. (C) and (D) show radiological examinations directly after implant removal, showing no significant loss of the sagittal or coronal balance. (E) and (F) demonstrate the radiological examinations during the follow-up examination (15 months after implant removal). No significant loss of the sagittal or coronal balance compared with the pre- and directly postoperative radiological examinations could be identified. 
Table 4. Detailed distribution of medical complications during hospital stay

\begin{tabular}{lc|} 
Variable & No. of complications \\
\hline None & 23 \\
\hline Pneumonia & 9 \\
\hline Anemia & 7 \\
\hline Cardiac decompensation & 5 \\
\hline Death & 4 \\
\hline Urinary tract infection & 4 \\
\hline Acute renal failure & 2 \\
\hline Sacral decubitus & 2 \\
\hline Myocardial infarction & 1 \\
\hline Anaphylactic shock & 1 \\
\hline Enterocolitis & 1 \\
\hline
\end{tabular}

In 11 patients (23.9\%), VAC therapy was used during the treatment period. Antibiotic sponges and vancomycin powder were used in two patients (4.3\%). In two patients (4.3\%), overlap plastic surgery (muscle flap) was necessary to achieve soft tissue coverage. The median number of operative interventions was three in the whole patient population.

\section{Hospitalization, complications, and outcome}

The mean length of hospital stay was $56.1 \pm 47.0$ days (range, 8-278 days). Postoperative complications occurred in 23 patients (50\%) (Table 4). During hospitalization, four patients (8.7\%) died of cardiopulmonary

Table 5. Statistical analysis of the demographics and clinical data in group 1 (implant retention) and group 2 (implant removal)

\begin{tabular}{|c|c|c|c|c|c|c|}
\hline \multirow{2}{*}{ Variable } & \multirow{2}{*}{ Category } & \multicolumn{2}{|c|}{ Group 1} & \multicolumn{2}{|c|}{ Group 2} & \multirow{2}{*}{$p$-value } \\
\hline & & No. of patients & Mean value & No. of patients & Mean value & \\
\hline \multirow[t]{2}{*}{ Sex } & Male & 10 & & 9 & & 0.430 \\
\hline & Female & 11 & & 16 & & \\
\hline Age (yr) & & & 65 & & 65 & 0.691 \\
\hline Segments with instrumentation & & & 7 & & 6 & 0.470 \\
\hline \multirow[t]{4}{*}{ ASA classification } & ASA 1 & 0 & & 2 & & 0.445 \\
\hline & ASA 2 & 8 & & 9 & & \\
\hline & ASA 3 & 8 & & 10 & & \\
\hline & ASA 4 & 5 & & 4 & & \\
\hline \multirow[t]{4}{*}{ Primary disease } & Neoplasm & 5 & & 2 & & 0.286 \\
\hline & Degeneration & 11 & & 16 & & \\
\hline & Deformity & 3 & & 3 & & \\
\hline & Trauma & 2 & & 4 & & \\
\hline \multirow[t]{2}{*}{ Infection timing } & Early & 18 & & 14 & & $0.031^{*}$ \\
\hline & Late & 3 & & 11 & & \\
\hline \multirow[t]{2}{*}{ High white blood cell count } & Yes & 7 & & 10 & & 0.667 \\
\hline & No & 12 & & 13 & & \\
\hline C-reactive protein level (mg/L) & & & 123 & & 150 & 0.225 \\
\hline \multirow[t]{2}{*}{ Infection type } & Monomicrobial & 11 & & 17 & & 0.377 \\
\hline & Polymicrobial & 9 & & 8 & & \\
\hline \multirow[t]{2}{*}{ Fever } & Yes & 4 & & 8 & & 0.371 \\
\hline & No & 16 & & 17 & & \\
\hline Length of hospitalization (day) & & & 43 & & 67 & $0.021^{*}$ \\
\hline \multirow[t]{2}{*}{ Complications } & Yes & 11 & & 12 & & 0.770 \\
\hline & No & 10 & & 13 & & \\
\hline
\end{tabular}

ASA, American Society of Anesthesiologists.

${ }^{*} p<0.05$; statistically significant. 
complications (pneumonia and cardiac decompensation). In 39 patients $(84.8 \%)$, infection was eradicated at a mean follow-up of 18.9 \pm 11.1 months (range, 12-60 months). Three patients $(6.5 \%)$ were lost to follow-up.

\section{Intergroup comparison}

Implant retention was possible in 21 patients $(45.7 \%)$, while implant removal was necessary in 25 patients (54.3\%). No intergroup differences were found in the demographic or clinical characteristics. The mean length of hospital stay was significantly longer in the implant removal group than in the implant retention group (implant retention group, 43 days; implant removal group, 67 days; $p=0.021$ ) (Table 5). Furthermore, the median number of surgical interventions was significantly higher in the implant retention group (two surgical interventions) than in the implant removal group (three surgical interventions) $(p=0.024)$. Implant removal was not associated with higher complication rates $(p=0.770)$. Three patients in the implant retention group and one patient in the implant removal died during hospitalization. Detailed intergroup comparison of the demographic, clinical, and outcome parameters is shown in Table 5. No differences in Gram staining were documented (Table 6). The mean follow-up period of the patients in the implant removal group was
$18.3 \pm 9.3$ months and that of the implant retention group was $19.7 \pm 13.4$ months. No statistical difference was found between the two groups $(p=0.703)$.

\section{Risk factors for implant removal}

Implant retention was possible in 18 cases (39\%) of early infection and in three cases $(6.5 \%)$ of late infection. A statistically significant correlation was found between implant removal and late infection ( $c=0.306, p=0.029)$. Two patients with late infection received continuous oral suppressive antibiotic therapy. No correlations were found between mono- or polymicrobial infection and implant removal ( $c=0.132, p=0.371)$. Moreover, no correlation was observed between primary disease $(c=0.229, p=0.466)$, localization of the instrumentation ( $c=0.280, p=0.865)$, or implant removal. Finally, CRP values and high WBC counts upon admission were not correlated with implant removal $(r=0.189, p=0.230$, and $p=0.667)$. Table 7 shows

Table 6. Gram stain distributions by group

\begin{tabular}{ccc} 
& No. of Gram positive & No. of Gram negative \\
Group 1 & 27 & 6 \\
Group 2 & 31 & 4 \\
\hline
\end{tabular}

Group 1, implant retention; Group 2, implant removal.

Table 7. Multiple regression analysis of the risk factors for implant removal

\begin{tabular}{|c|c|c|c|c|c|c|}
\hline \multirow{2}{*}{ Variable } & \multicolumn{2}{|c|}{$\begin{array}{l}\text { Not standardized } \\
\text { coefficients }\end{array}$} & \multirow{2}{*}{$\begin{array}{c}\text { Standardized } \\
\text { regression coefficients } \\
\text { Beta }\end{array}$} & \multirow{2}{*}{$t$-value } & \multirow{2}{*}{$p$-value } & \multirow{2}{*}{$\begin{array}{l}95 \% \text { Confidence } \\
\text { interval }\end{array}$} \\
\hline & $\begin{array}{l}\text { Regression } \\
\text { coefficient }\end{array}$ & $\begin{array}{l}\text { Standard } \\
\text { error }\end{array}$ & & & & \\
\hline \multicolumn{7}{|l|}{ Preoperative data } \\
\hline Sex (male vs. female) & 0.447 & 0.212 & 0.437 & 2.114 & 0.051 & 0.008 to 0.886 \\
\hline Age & 0.005 & 0.008 & 0.142 & 0.599 & 0.555 & -0.012 to 0.021 \\
\hline Segments of instrumentation & -0.054 & 0.049 & -0.244 & -1.094 & 0.286 & $-0,156$ to 0.048 \\
\hline Region of spinal instrumentation & 0.051 & 0.047 & 0.216 & 1.075 & 0.294 & -0.047 to 0.148 \\
\hline Primary disease & 0.112 & 0.119 & 0.189 & 0.946 & 0.354 & -0.134 to 0.359 \\
\hline American Society of Anesthesiologists classification & -0.022 & 0.124 & -0.036 & -0.176 & 0.862 & -0.278 to 0.235 \\
\hline Infection timing (early vs. late) & 0.445 & 0.188 & 0.423 & 2.362 & $0.026^{*}$ & 0.057 to 0.833 \\
\hline Fever (yes vs. no) & 0.118 & 0.234 & 0.105 & 0.504 & 0.619 & -0.367 to 0.602 \\
\hline High white blood cell count (yes vs. no) & -0.177 & 0.281 & -0.175 & -0.630 & 0.535 & -0.759 to 0.406 \\
\hline C-reactive protein & 0.000 & 0.001 & -0.047 & -0.180 & 0.859 & -0.003 to 0.002 \\
\hline Infection type (mono- vs. polymicrobial) & -0.443 & 0.215 & -0.439 & -2.056 & 0.052 & -0.890 to 0.004 \\
\hline
\end{tabular}

$p<0.05$; statistically significant. 
the results of the multiple regression analysis. Late infections were identified as risk factors of implant removal.

\section{Discussion}

Surgical site infection following spinal instrumentation leads to high morbidity and mortality rates and higher healthcare costs $[14,15]$. In this sense, proper treatment of the infection is essential for patient recovery and eradication of infection. Infections of long-segment instrumentation can be related to high rates of failure to retain the implant, leading to implant removal [16]. Considering that implant removal is associated with loss of correction and degenerative spine conditions, instrumentation length plays an important role in the decision-making process for the specific surgical treatment of the infection [12]. In addition, implant removal and re-instrumentation are associated with high intraoperative blood loss and high complication rates [17].

The main findings of the study revealed that implantassociated infections of long-segment spinal instrumentations are associated with high postoperative complication rates $(50 \%)$ and high mortality rates $(8.7 \%)$. These findings correlate with previous findings. Casper et al. [15] reported significantly higher mortality rates in patients with postoperative spinal infections than in those without postoperative spinal infections. In another retrospective review of 28 cases of infected posterior spinal instrumentation, the postoperative complication rate was $46 \%$ [17]. However, the complication rates were similar in implant retention and removal in our study. In this sense, a higher incidence of complications was not correlated with implant removal.

With regard to surgical implant management, the findings of the present study revealed that retention was possible in $45.7 \%$ of the patients. Kim et al. [18] reported that implant removal for the treatment of infected instrumented spinal fusion could lead to satisfactory clinical outcomes. However, the authors reported loss of lordosis, pseudarthrosis, and disc space collapse after implant removal. In this study, the instrumented fusion length was 1-4 lumbar segments [18]. Hedequist et al. [10] advocated that implant removal led to good clinical outcomes in the treatment of delayed instrumented spinal infection. A further study investigating clinical and radiological outcomes following spinal instrumentation removal in cases with late infection in posterior fusion in scoliosis reported suf- ficient clinical outcomes. However, implant removal was associated with significant loss of correction. Re-instrumentation was necessary to preserve correction [11]. According to our results, re-instrumentation was necessary in $39.1 \%$ of the patients due to the absence of bony fusion and presence of segmental instability. Complete implant removal without re-instrumentation was only possible in $4.3 \%$ of the patients, neither of whom demonstrated loss of correction in the sagittal and coronal planes.

In a multicenter retrospective survey, Tsubouchi et al. [19] reported an implant retention rate of $60 \%$ among infected spinal instrumentation cases. According to the authors, implant retention was more likely achieved in the cervical than in the thoracolumbar spine [19]. This aspect could not be observed in our study. Our results suggested that localization of the instrumentation was not correlated with implant retention or removal. Interestingly, NúñezPereira et al. [16] showed a lower implant retention rate (20.9\%) than our implant retention rate $(45.7 \%)$ in deep infection following spinal instrumentation.

Based on our study results, late infection was a risk factor for implant removal. Tsubouchi et al. [19] reported that implant retention was not possible in late infection ( $>90$ days after the index operation). In our study, implant retention in late infection was possible in three cases (6.5\%). Further risk factors for implant removal described by Tsubouchi et al. [19] were high blood loss at the index surgery, delayed revision surgery, and delayed administration of effective antibiotics. In this study, the mean length of the instrumentation was three segments.

Implant removal can be necessary for the treatment of implant-associated spinal infections because of the presence of biofilm on the artificial implant [20]. The formation of bacterial biofilm reduces the susceptibility of bacteria to antibiotics, and the infection can remain active despite proper antibiotic treatment [8]. The implant surface stimulates the adhesion of microorganisms and formation of biofilm [9]. In early infection, biofilm formation can be prevented by wide surgical debridement and early antibiotic treatment. However, in late infection, the instrumentation is typically covered with biofilm, leading to resistance to antimicrobial agents and inhibited host defenses [5,21].

In the surgical management of the implant, no influence of pathogens was found in our study. Polymicrobial infections were not associated with implant removal. Moreover, Gram staining of pathogens did not show any correlation 
with implant retention or removal. The most common pathogens were S. epidermidis and methicillin-susceptible S. aureus. Low virulent pathogens tend to cause late implant-associated infections due to the unclear clinical course of the infection [5]. According to this observation, implant-associated infections with low virulent pathogens should be associated with higher implant removal rates. However, in our cohort, low virulent pathogens such as $S$. epidermidis, Staphylococcus hominis, and S. haemoliticus were equally distributed in both groups. Furthermore, $P$. acnes was isolated more frequently in the implant retention group than in the implant removal group (4:1). In this regard, the virulence of the pathogen did not influence the surgical management of implant-associated infections in our cohort.

Our results indicate that implant removal was associated with late infection. Several studies advocated implant removal in late implant-associated infections $[5,11,20,22]$. In this sense, implant removal should be considered for the treatment of implant-associated infections of longsegment instrumentation. The results of our study showed that implant removal could lead to eradication of the infection without higher complication and mortality rates than implant retention. Implant removal was related with longer hospitalization than implant retention. A possible reason for this could be failure to retain the implant, leading to more surgical interventions and longer hospitalization. This aspect could be observed in our study. The number of surgical interventions was significantly higher in the implant removal group than in the implant retention group. In this sense, the attempt to preserve the implant leads to more surgical interventions and longer hospitalization. Thus, earlier implant removal in late implant-associated infections of long-segmental spinal instrumentations could reduce the number of surgical interventions and length of hospitalization.

In implant retention or re-instrumentation after implant removal, antibiotic treatment with biofilm-active antimicrobial agents should be prescribed to prevent biofilm formation on the implants. Nevertheless, implant-associated infections of long-segment spinal instrumentation should be treated in cooperation with infectiologists and microbiologists to ensure the development of a reasonable treatment strategy. As shown in our study, adapting a reasonable treatment protocol can lead to eradication of the implant-associated infection of long-segment spinal instrumentation. Continuous oral suppressive antibiotic therapy could be a treatment option of persistent spinal infection and surgical inability for complete removal of the spinal instrumentation, e.g., multisegmental cementaugmented pedicle screws or poor clinical condition of the patient. This treatment option has been reported for patients with an implant-associated infection in which surgical intervention is contraindicated [23].

This study has several limitations. First, selection bias is possible because of its retrospective design. Second, implant retention or removal cannot be decided for all patients despite the standardized treatment protocol of the osteomyelitis board. In this sense, selection bias by the surgeon's preference could affect the decisions of implant surgical management. Finally, the relatively small patient cohort reduces the statistical strength of the data.

\section{Conclusions}

Implant-associated infections of long-segment spinal instrumentations are associated with high complication and mortality rates. In our patient cohort, implant retention was possible in $45.7 \%$ of the patients. In late infections, implant removal was necessary to eradicate the infection. Therefore, based on the findings of this study, implant removal can be recommended in late implant-associated infections of spinal instrumentations.

\section{Conflict of Interest}

No potential conflict of interest relevant to this article was reported.

\section{Author Contributions}

Stavros Oikonomidis contributed to the writing of the paper, to searching for references and data analysis. Lisa Altenrath and Leonard Westermann contributed to the data collection and analysis. Jan Bredow contributed to writing of the paper. Peer Eysel contributed to the study design. Max Joseph Scheyerer contributed to data analysis, writing of the paper and supervised the project. All authors reviewed and approved the final submitted version of the paper.

\section{References}

1. Kasliwal MK, Tan LA, Traynelis VC. Infection with 
spinal instrumentation: review of pathogenesis, diagnosis, prevention, and management. Surg Neurol Int 2013;4(Suppl 5):S392-403.

2. Chaichana KL, Bydon M, Santiago-Dieppa DR, et al. Risk of infection following posterior instrumented lumbar fusion for degenerative spine disease in 817 consecutive cases. J Neurosurg Spine 2014;20:45-52.

3. Fang A, Hu SS, Endres N, Bradford DS. Risk factors for infection after spinal surgery. Spine (Phila $\mathrm{Pa}$ 1976) 2005;30:1460-5.

4. Liu JM, Deng HL, Chen XY, et al. Risk factors for surgical site infection after posterior lumbar spinal surgery. Spine (Phila Pa 1976) 2018;43:732-7.

5. Viola RW, King HA, Adler SM, Wilson CB. Delayed infection after elective spinal instrumentation and fusion: a retrospective analysis of eight cases. Spine (Phila Pa 1976) 1997;22:2444-50.

6. Lall RR, Wong AP, Lall RR, Lawton CD, Smith ZA, Dahdaleh NS. Evidence-based management of deep wound infection after spinal instrumentation. J Clin Neurosci 2015;22:238-42.

7. Darouiche RO. Antimicrobial approaches for preventing infections associated with surgical implants. Clin Infect Dis 2003;36:1284-9.

8. Gracia E, Fernandez A, Conchello P, et al. Adherence of Staphylococcus aureus slime-producing strain variants to biomaterials used in orthopaedic surgery. Int Orthop 1997;21:46-51.

9. Hickok NJ. What are biofilms? Spine (Phila Pa 1976) 2018;43:S7-8.

10. Hedequist D, Haugen A, Hresko T, Emans J. Failure of attempted implant retention in spinal deformity delayed surgical site infections. Spine (Phila Pa 1976) 2009;34:60-4.

11. Muschik M, Luck W, Schlenzka D. Implant removal for late-developing infection after instrumented posterior spinal fusion for scoliosis: reinstrumentation reduces loss of correction: a retrospective analysis of 45 cases. Eur Spine J 2004;13:645-51.

12. Ho C, Skaggs DL, Weiss JM, Tolo VT. Management of infection after instrumented posterior spine fu- sion in pediatric scoliosis. Spine (Phila Pa 1976) 2007;32:2739-44.

13. Mangram AJ, Horan TC, Pearson ML, Silver LC, Jarvis WR. Guideline for prevention of surgical site infection, 1999. Hospital Infection Control Practices Advisory Committee. Infect Control Hosp Epidemiol 1999;20:250-78.

14. Calderone RR, Garland DE, Capen DA, Oster H. Cost of medical care for postoperative spinal infections. Orthop Clin North Am 1996;27:171-82.

15. Casper DS, Zmistowski B, Hollern DA, et al. The effect of postoperative spinal infections on patient mortality. Spine (Phila Pa 1976) 2018;43:223-7.

16. Nunez-Pereira S, Pellise F, Rodriguez-Pardo D, et al. Implant survival after deep infection of an instrumented spinal fusion. Bone Joint J 2013;95-B:1121-6.

17. Andras LM, Joiner ER, Skaggs K, et al. Removal of infected posterior spinal implants: be prepared to transfuse. Spine Deform 2016;4:283-7.

18. Kim JI, Suh KT, Kim SJ, Lee JS. Implant removal for the management of infection after instrumented spinal fusion. J Spinal Disord Tech 2010;23:258-65.

19. Tsubouchi N, Fujibayashi S, Otsuki B, et al. Risk factors for implant removal after spinal surgical site infection. Eur Spine J 2018;27:2481-90.

20. Richards BS. Delayed infections following posterior spinal instrumentation for the treatment of idiopathic scoliosis. J Bone Joint Surg Am 1995;77:524-9.

21. Costerton JW, Stewart PS, Greenberg EP. Bacterial biofilms: a common cause of persistent infections. Science 1999;284:1318-22.

22. Kowalski TJ, Berbari EF, Huddleston PM, Steckelberg JM, Mandrekar JN, Osmon DR. The management and outcome of spinal implant infections: contemporary retrospective cohort study. Clin Infect Dis 2007;44:913-20.

23. Leijtens B, Weerwag L, Schreurs BW, Kullberg BJ, Rijnen W. Clinical outcome of antibiotic suppressive therapy in patients with a prosthetic joint infection after hip replacement. J Bone Jt Infect 2019;4:268-76. 